\title{
VLBA IMAGING POLARIMETRY OF ACTIVE GALACTIC NUCLEI: AN AUTOMATED APPROACH
}

\author{
G. B. Taylor, ${ }^{1,2}$ C. D. Fassnacht, ${ }^{3}$ L. O. Suoumerman, ${ }^{2}$ S. T. Myers, ${ }^{2}$ J. S. Ulvestad, ${ }^{2}$ R. C. Walker, ${ }^{2}$ \\ E. B. Fomalont, ${ }^{2}$ T. J. Pearson, ${ }^{4}$ A. C. S. Readhead, ${ }^{4}$ N. Gehrels,${ }^{5}$ and P. F. Michelson ${ }^{6}$ \\ Received 2005 January 17; accepted 2005 March 9
}

\begin{abstract}
We present full polarization Very Long Baseline Array (VLBA) observations at 5 and $15 \mathrm{GHz}$ of 24 compact active galactic nuclei (AGNs). These sources were observed as part of a pilot project to demonstrate the feasibility of conducting a large VLBI survey to further our understanding of the physical properties and temporal evolution of AGN jets. The sample is drawn from the Cosmic Lens All-Sky Survey (CLASS) where it overlaps with the Sloan Digital Sky Survey at declinations north of $15^{\circ}$. There are 2100 CLASS sources brighter than 50 mJy at $8.4 \mathrm{GHz}$, of which we have chosen 24 for this pilot study. All 24 sources were detected and imaged at $5 \mathrm{GHz}$ with a typical dynamic range of 500:1, and 21 of 24 sources were detected and imaged at $15 \mathrm{GHz}$. Linear polarization was detected in eight sources at both 5 and $15 \mathrm{GHz}$, allowing for the creation of Faraday rotation measure (RM) images. The core RMs for the sample were found to have an average absolute value of $390 \pm 100 \mathrm{rad} \mathrm{m}^{-2}$. We also present the discovery of a new compact symmetric object, J08553 +5751 . All data were processed automatically using pipelines created or adapted for the survey.
\end{abstract}

Subject headings: catalogs — galaxies: active — galaxies: jets — galaxies: nuclei — radio continuum: galaxies — surveys - techniques: polarimetric

Online material: color figures

\section{INTRODUCTION}

Among astronomical observations, very long baseline interferometry (VLBI) imaging is unique in providing details about parsec-scale structures in cosmologically distant objects. The Pearson-Readhead (1988, hereafter PR) survey revealed a wide range in morphologies and paved the way toward motion and variability studies. Subsequent VLBI surveys such as the CaltechJodrell Bank Flat spectrum survey (Taylor et al. 1996, hereafter CJF), have imaged $\sim 300$ sources at $5 \mathrm{GHz}$, of which more than half (177) were observed in full polarization (Pollack et al. 2003). The VLBA Calibrator Survey (Beasley et al. 2002, hereafter VCS) included $\sim 2000$ sources, but at the expense of limited sensitivity and $(u, v)$ coverage since the driving goals were an astrometric grid of phase-referencing calibrators, and not high-quality imaging.

A new survey of $\sim 1000$ sources with full polarization at 5 and $15 \mathrm{GHz}$ would provide an unparalleled combination of size, depth, and polarization information. The large sample of highquality data sets, especially when combined with surveys at other wavelengths, would provide an excellent resource for studying the physics, environments, and evolution of active galactic nuclei (AGNs). The key areas of study for a large survey are as follows.

Gamma-ray AGNs.-This new Very Long Baseline Array (VLBA) survey, combined with the survey data from the GammaRay Large Area Space Telescope (GLAST) mission (Gehrels \&

\footnotetext{
1 Kavli Institute of Particle Astrophysics and Cosmology, Menlo Park, CA 94025.

2 National Radio Astronomy Observatory, P.O. Box O, Socorro, NM 87801.

3 Department of Physics, University of California, Davis, 1 Shields Avenue, Davis, CA 95616.

4 Owens Valley Radio Observatory, California Institute of Technology, Pasadena, CA 91125.

5 NASA Goddard Space Flight Center, Greenbelt, MD 20771.

6 Department of Physics, Stanford University, 382 Via Pueblo Mall, Stanford, CA 94305.
}

Michelson 1999), should revolutionize our understanding of AGN jet physics. After launch in 2007, GLAST will perform an all-sky survey 30 times more sensitive than that of EGRET. The number of AGNs expected to be detected is $~ 3000-4500$, although variability, especially in the gamma-ray properties, may strongly influence these estimates (Vercellone et al. 2004). Correlations with milliarcsecond polarimetry are key to identifying and understanding the gamma-ray sources. Extrapolating from the correlation between $5 \mathrm{GHz}$ radio luminosity and peak gamma-ray emission (Mattox et al. 1997), we expect that most of these objects will be compact, flat-spectrum sources with $5 \mathrm{GHz}$ flux densities over $30 \mathrm{mJy}$. EGRET results and theoretical studies indicate that gamma-ray emission emanates from jet outflows (e.g., Dermer \& Schlickeiser 1994), where highenergy nonthermal electrons accelerated in shocks are thought to Compton-upscatter soft photons. Milliarcsecond radio imaging can reveal the detailed morphological structure of the core and outflow regions. With a large number of sources, correlations between radio morphology and gamma-ray spectral properties will be determined with high significance, thus providing constraints on models for baryonic acceleration and the microphysics of electron-proton coupling. In addition, the improved error box of GLAST ( $\sim$ arcminutes) combined with the VLBA imaging may solve the mystery of the unidentified high-latitude EGRET sources.

Jet magnetic fields.--Recently, Pollack et al. (2003) have found a tendency for the parsec-scale cores of quasars to have a magnetic field aligned in the direction of the jet, which can constrain jet-launching/collimation models (e.g., Meier et al. 2001). Similar observations of BL Lacs have suggested that their magnetic fields are oriented perpendicular to the jets (e.g., Gabuzda et al. 2000). Unfortunately, the number of BL Lacs and galaxies with good polarization data are too small for a meaningful comparison between different classes of objects. The 1000 source survey will address this problem by providing $\geq 100$ objects in each class. 
AGN environments. - The survey data will be used to study propagation effects local to the jet, including free-free absorption in sources with counterjets and Faraday rotation measures (RMs) toward all sources with detected polarization. Typical quasars have RMs that are surprisingly large $\left(>1000 \mathrm{rad} \mathrm{m}^{-2}\right.$; Taylor 1998) and time variable (Zavala \& Taylor 2001). Knowing the $\mathrm{RM}$ is also critical in order to make sense of the jet properties, as otherwise the RM will tend to "smear out" intrinsic relationships.

AGN evolution.-Compact symmetric objects (CSOs), by virtue of their small sizes $(<1 \mathrm{kpc})$, are thought to be examples of young $\left(10^{3}-10^{4} \mathrm{yr}\right)$ radio sources that may evolve to become eventually the well-known FR II-type radio sources (Readhead et al. 1996). Only small numbers of CSOs are known ( 40; Peck $\&$ Taylor 2000), limiting our ability to study radio sources at early times in their evolutionary history. A large VLBA survey would approximately double the number of confirmed CSOs.

Here we present the results from a feasibility study for a possible VLBI Imaging and Polarimetry Survey (VIPS). While not a complete sample in itself, this pilot study of 24 AGNs was chosen to be a representative selection of strong and weak sources and presents a wealth of data on this modest sample. This includes one new CSO $(\mathrm{J} 08553+5751)$ and the first Faraday rotation measure observations of a sample of faint sources. We have also developed and made available new pipelines for calibration and imaging that allow rapid reduction of the VLBA data to finished products. Throughout this discussion, we assume $H_{0}=70 \mathrm{~km}$ $\mathrm{s}^{-1} \mathrm{Mpc}^{-1}, \Omega_{M}=0.27$, and $\Omega_{\Lambda}=0.73$.

\section{SAMPLE DEFINITION}

To facilitate multiwavelength science, all targets lie within the $\pi$ sr region of the North Galactic Cap and the equatorial strips to be covered by the Sloan Digital Sky Survey (SDSS; Abazajian et al. 2004, 2005). Thus, multicolor imaging (UV to near-IR) will be available for almost all targets, and spectroscopic information will be present for the optically bright targets (perhaps 33\%). For optically faint sources, photometric redshifts may be estimated from the ugriz imaging data (Weinstein et al. 2004). In this region there are 2100 flat-spectrum, compact (size $<200$ mas), and relatively bright $\left(S_{8.5 \mathrm{GHz}}>50 \mathrm{mJy}\right)$ sources above $15^{\circ}$ declination in Cosmic Lens All-Sky Survey (CLASS) (Myers et al. 2003), a VLA survey of $\sim 12,000$ flat-spectrum radio sources at 200 mas resolution. From this number we have selected a sample of 1000 sources never before imaged at high dynamic range with VLBI. The declination limit of $15^{\circ}$ is necessary to insure uniformly good radio imaging quality. The lower limit of $50 \mathrm{mJy}$ has been chosen in order to have sufficient SNR $(>1.5$ estimated at $15 \mathrm{GHz}$ ) on all compact sources with a spectral index $>-0.5$ to permit self-calibration within the coherence time at both 5 and $15 \mathrm{GHz}$, where the spectral index, $\alpha$, is defined as $S_{\nu} \propto \nu^{\alpha}$. Heavily resolved sources (sizes $>20$ mas) may still be undetectable, especially at $15 \mathrm{GHz}$, where $25 \mathrm{mJy}$ is needed on scales $<20$ mas for self-calibration. We note that there is an implicit avoidance of the Galactic plane in our selection since CLASS was selected to have $|b|>10^{\circ}$. CLASS also had a spectral selection of $\alpha>-0.5$ from the parent Green Bank Survey (Gregory et al. 1996, hereafter GB6) at $4.85 \mathrm{GHz}$ and the NRAO VLA Sky Survey (Condon et al. 1998, hereafter NVSS) at $1.4 \mathrm{GHz}$. A similar spectral selection was employed for the CJF survey to obtain a high success rate with VLBI imaging.

We note that there should be $\sim 1000$ GLAST sources found in the area covered by VIPS. Since this is comparable to the number of VIPS sample members in the same region, and compact flat-spectrum radio emission is believed to be a strong predictor of gamma-ray emission (Mattox et al. 2001; Sowards-Emmerd et al. 2003), a milliarcsecond radio imaging survey of the VIPS sample may be important for the scientific interpretation of the GLAST all-sky survey (as discussed in $\S 1$ ).

Here we present observations of 24 sources (see Table 1) selected within the first and second data release areas of SDSS, and representative of the VLBA VIPS sample as a whole. The integrated flux densities of the $8.4 \mathrm{GHz}$ VLA parent sample range from $52 \mathrm{mJy}$ for $\mathrm{J} 08585+5552$ to $850 \mathrm{mJy}$ for J08546+5757 (JVAS $0850+581$ ). A total of $8,7,5$, and 4 sources were selected from the ranges 50-100, 100-200, 200-400, and >400 mJy.

\section{OBSERVATIONS AND ANALYSIS}

The observations were carried out at 5 and $15 \mathrm{GHz}$ on 2004 March 14, 2004 March 15, 2004 June 28, and 2004 August 18, using the VLBA of the NRAO. ${ }^{7}$ Each observing session lasted for $12 \mathrm{hr}$. The 5 and $15 \mathrm{GHz}$ frequency bands were interleaved in time, with 24 minutes scheduled on each target source at $5 \mathrm{GHz}$ and 72 minutes on source at $15 \mathrm{GHz}$. Allowing for telescope slews and other overhead ( 6 minutes on average) the time needed for each source was $1.7 \mathrm{hr}$. In order to improve $(u, v)$ coverage and to allow for rotation measure determinations, we spread the frequencies out over the $500 \mathrm{MHz}$ instantaneous tuning range of the VLBA as follows: $5 \mathrm{GHz}$ refers to $4607,4677,4992$, and $5097 \mathrm{MHz}$ at band center, while $15 \mathrm{GHz}$ refers to 14904, 14970, 15267 , and $15366 \mathrm{MHz}$ at band center. Each frequency was observed with $4 \mathrm{MHz}$ bandwidth in each of right circular and left circular polarization. The only significant loss of data were the loss of 98 minutes because of a false fire alarm at the Hancock, NH VLBA station on 18 August. Rain at some sites increased system temperatures and reduced sensitivity, especially at $15 \mathrm{GHz}$, in all four runs.

Amplitude calibration was derived using measurements of the system temperatures and antenna gains at 4992 and $15366 \mathrm{MHz}$, which are close to the continuum default frequencies of 4999 and $15369 \mathrm{MHz}$. Fringe-fitting was performed with the AIPS task FRING on the strong calibrator 3C 279. Feed polarizations of the antennas were determined at 5 and $15 \mathrm{GHz}$ using the AIPS task LPCAL and the unpolarized source OQ208. An initial phase self-calibration was performed for each source using a point source model - no phase referencing to nearby calibrators was used, as this would significantly reduce the observing efficiency and is unnecessary for such strong sources.

Absolute electric vector position angle (EVPA) calibration was determined by using the EVPA's of 3C 279, J0854+2006, and $\mathrm{J} 1310+3220$ as listed in the VLA Monitoring Program ${ }^{8}$ (Taylor \& Myers 2000). Agreement in the EVPA correction determined from these calibrators was generally better than 3 degrees for observations within a few days. All observations of EVPAs for the target sources have been corrected for Faraday rotation as determined from the observations.

For each source, the $15 \mathrm{GHz}$ data were tapered to produce an image at comparable resolution to the full resolution $5 \mathrm{GHz}$ image. For simplicity all sources were restored with a fixed beam size of $3.2 \times 1.6 \mathrm{mas}$ in position angle $0^{\circ}$ at each frequency. The two images were then combined to generate a spectral index map. It is important to note that spectral index maps made from two data sets with substantially different $(u, v)$ coverages may

\footnotetext{
7 The National Radio Astronomy Observatory is a facility of the National Science Foundation operated under cooperative agreement by Associated Universities, Inc.

${ }^{8}$ See http://www.vla.nrao.edu/astro/calib/polar/.
} 
TABLE 1

VIPS PILOT SAMPLE

\begin{tabular}{|c|c|c|c|c|c|c|c|c|}
\hline $\begin{array}{c}\text { Source Name } \\
\text { (1) }\end{array}$ & $\begin{array}{l}\text { Alternate } \\
\text { Name } \\
(2)\end{array}$ & $\begin{array}{l}\text { R.A. } \\
\text { (J2000) } \\
\text { (3) }\end{array}$ & $\begin{array}{l}\text { Decl. } \\
\text { (J2000) } \\
\text { (4) }\end{array}$ & $\begin{array}{c}8 \mathrm{GHz} \\
S \\
(5)\end{array}$ & $\begin{array}{l}\text { ID } \\
(6)\end{array}$ & $\begin{array}{l}M_{r} \\
\text { (7) }\end{array}$ & $\begin{array}{c}z \\
(8)\end{array}$ & $\begin{array}{c}\text { Date } \\
(9)\end{array}$ \\
\hline J08474+5723 ............ & $0843+575$ & 084728.0579 & 572338.349 & 240 & $\ldots$ & & $\ldots$ & $\mathrm{C}$ \\
\hline $\mathrm{J} 08490+5603 \ldots \ldots \ldots \ldots \ldots$ & & 084900.8546 & 560350.122 & 70 & Q & 21.267 & $\ldots$ & A \\
\hline J08499+5108................ & & 084957.9836 & 510828.997 & 325 & $\mathrm{Q}$ & 18.194 & 0.584 & A \\
\hline J08507+5159 ................ & & 085042.2482 & 515911.674 & 112 & $\mathrm{Q}$ & 18.961 & 1.892 & A \\
\hline J08546+5757 ............... & $0850+581$ & 085441.9973 & 575729.927 & 890 & $\mathrm{Q}$ & 17.739 & 1.318 & $\mathrm{C}$ \\
\hline J08553+5751 ................. & $0851+580$ & 085521.3558 & 575144.091 & 160 & $\mathrm{G}$ & 19.843 & $\ldots$ & $\mathrm{C}$ \\
\hline J08575+5827 ................ & & 085731.4511 & 582722.538 & 88 & Q & 22.082 & $\ldots$ & B \\
\hline 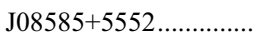 & & 085830.4786 & 555241.302 & 52 & $\mathrm{G}$ & 18.473 & $\ldots$ & B \\
\hline J09030+4651 ................ & $0859+470$ & 090303.9901030 & 465104.13753 & 963 & Q & 18.989 & 1.47 & B \\
\hline J09470+5907 ................ & & 094704.8631 & 590741.467 & 70 & $\ldots$ & $\ldots$ & $\ldots$ & $\mathrm{D}$ \\
\hline J09493+6039 ................ & & 094920.2283 & 603922.871 & 325 & Q & 19.917 & $\ldots$ & $\mathrm{D}$ \\
\hline J09496+5819 ……....... & & 094939.8149 & $\begin{array}{lll}58 & 19 & 12.933\end{array}$ & 112 & Q & 21.308 & $\ldots$ & $\mathrm{D}$ \\
\hline $\mathrm{J} 14142+4554 \ldots \ldots \ldots \ldots \ldots$ & $1412+461$ & 141414.8535061 & 455448.65427 & 80 & G & 20.158 & $\ldots$ & B \\
\hline $\mathrm{J} 15406+5803 \ldots \ldots \ldots \ldots . .$. & & 154037.5779 & 580334.398 & 71 & Q & 18.549 & 1.25 & A \\
\hline $\mathrm{J} 15450+5135 \ldots \ldots \ldots \ldots$ & $1543+517$ & 154502.8222 & 513500.874 & 635 & $\mathrm{Q}$ & 17.552 & 1.93 & A \\
\hline $\mathrm{J} 15457+5400 \ldots \ldots \ldots \ldots$ & & 154543.8287 & 540042.764 & 145 & Q & 19.827 & $\ldots$ & A \\
\hline $\mathrm{J} 15465+5146 \ldots \ldots \ldots \ldots$ & & 154633.6208 & 514645.455 & 90 & $\mathrm{Q}$ & 19.943 & $\ldots$ & $\mathrm{B}$ \\
\hline $\mathrm{J} 15521+5552 \ldots \ldots \ldots \ldots \ldots$ & & $1552 \quad 10.8942$ & 555243.211 & 131 & $\mathrm{Q}$ & 20.688 & $\ldots$ & B \\
\hline J16445+3916 ............... & $1642+393$ & 164434.4775 & 391604.915 & 635 & Q & 19.808 & 1.583 & $\mathrm{D}$ \\
\hline $\mathrm{J} 16469+4059 \ldots \ldots \ldots \ldots$ & $1645+410$ & 164656.8603 & 405917.167 & 71 & $\mathrm{Q}$ & 19.252 & $\ldots$ & $\mathrm{D}$ \\
\hline $\mathrm{J} 16484+4104 \ldots \ldots \ldots \ldots \ldots$ & $1646+411$ & 164829.2622 & 410405.558 & 210 & Q & 18.741 & 0.852 & $\mathrm{C}$ \\
\hline $\mathrm{J} 16525+4013 \ldots \ldots \ldots \ldots$ & & 165233.2136 & 401358.339 & 100 & $\mathrm{Q}$ & 20.465 & $\ldots$ & $\mathrm{C}$ \\
\hline $\mathrm{J} 16529+3902 \ldots \ldots \ldots \ldots \ldots$ & $1651+391$ & 165258.5096 & 390249.807 & 330 & Q & 21.341 & $\ldots$ & $\mathrm{C}$ \\
\hline $\mathrm{J} 16542+3950 \ldots \ldots \ldots \ldots \ldots$ & & 165412.7223 & 395005.681 & 145 & $\mathrm{Q}$ & 19.948 & $\ldots$ & $\mathrm{D}$ \\
\hline
\end{tabular}

Notes.- (Col. [1]) J2000 source name in the IAU format HHMMd+DDMM; (col. [2]) alternate name; (cols. [3] and [4]) right ascension and declination in J2000 coordinates from CLASS ( Myers et al. 2003), except for 1412+461 and 1543+517 taken from the International Coordinate Reference Frame (ICRF; Ma et al. 1998) (units of right ascension are hours, minutes, and seconds, and units of declination are degrees, arcminutes, and arcseconds); (col. [5]) Flux density at $8.4 \mathrm{GHz}$ from CLASS; (col. [6]) optical host galaxy identification; (col. [7]) optical magnitude in $r$ band; (col. [8]) spectroscopic redshift from SDSS; (col. [9]) date of observation, (A) 2004 March 14, (B) 2004 March 15, (C) 2004 June 28, and (D) 2004 August 18.

suffer from significant systematic errors, especially in regions of extended emission. Polarization images were likewise made at matching resolutions in order to make rotation measure (RM) images. All calibration and imaging was done semiautomatically using pipelines written in AIPS (Greisen 2003) and Difmap (Shepherd 1997). These pipelines can be found at the VIPS Web site. ${ }^{9}$

For 23 out of 24 sources the automatic imaging scripts were able to produce an image with the expected noise and a good fit $(\sigma)$ between the source model and the data. The parameter $\sigma$ is the square root of the squared difference between the data and the model divided by the individual variances implied by the visibility weights. This is close to the square root of the reduced $\chi^{2}$, except that a small change in the number of degrees of freedom due to amplitude self-calibration has not been taken into account. This simplification is generally benign and will only result in a $\sim 10 \%$ reduction of $\sigma$ for sources strong enough to amplitude self-calibrate (peak flux density brighter than $0.3 \mathrm{Jy}$ at $5 \mathrm{GHz}$ or $0.5 \mathrm{Jy}$ at $15 \mathrm{GHz}$ ). The value of $\sigma$ indicates the agreement obtained between the model and data in the selfcalibration process with values near unity indicating good agreement. In one case a poor fit $(\sigma>1.4)$ was found to be due to bad data during 5 minutes from the Los Alamos, NM station. It is worth noting that in our initial automatic imaging script we used a field size of $64 \times 64$ mas, and this resulted in poor fits for two sources with emission beyond the edge of the field. Judicial manual editing was done to solve the first problem and imag-

\footnotetext{
${ }^{9}$ See http://www.aoc.nrao.edu/ gtaylor/VIPS/.
}

ing all sources with an initial field size of $128 \times 128$ mas then provided good fits for all 24 sources. The final images have rms noise values within $30 \%$ of the predicted thermal noise.

The observing efficiency of $1.7 \mathrm{hr}$ per source could be improved to $1.5 \mathrm{hr}$ per source without loss of sensitivity if the observing runs were extended to $24 \mathrm{hr}$. This could be done since the number of calibrator scans is the same for a $24 \mathrm{hr}$ run as it is for a $12 \mathrm{hr}$ run. The fraction of time spent on calibrators for each of the $12 \mathrm{hr}$ observing runs in the pilot project was $15 \%$.

\section{RESULTS}

Figure 1 displays total intensity images for all sources in the pilot project at 5 and $15 \mathrm{GHz}$. Contours are drawn starting at $0.8 \mathrm{mJy}$ beam $^{-1}$. Further details can be found in Tables 2 and 3 . All 24 sources were detected and imaged at $5 \mathrm{GHz}$. At $15 \mathrm{GHz}$ there was no detection for J08553+5751 = JVAS 0851+580 (see $\S 4.2 .1$ ), J08585+5552, or J15406+5803. Since no observations were carried out with phase-referencing, sources with a flux density less than $25 \mathrm{mJy}$ at $15 \mathrm{GHz}$ were too weak to self-calibrate. The lack of detections is due to a combination of moderately steep-spectrum sources and the lower sensitivity at $15 \mathrm{GHz}$ within the coherence time used for self-calibration of the phases. The sensitivity limit for detection was a flux density of $10 \mathrm{mJy}$ at $5 \mathrm{GHz}$ and $25 \mathrm{mJy}$ at $15 \mathrm{GHz}$.

Linear polarization was detected in 8 of 24 sources at both 5 and $15 \mathrm{GHz}$, and at $15 \mathrm{GHz}$ only in the source $\mathrm{J} 16542+3950$. In order to increase the SNR in the polarization images the observations at 4607 and $4677 \mathrm{MHz}$ were combined, as were those at 4992 and $5007 \mathrm{MHz}, 14904$ and $14970 \mathrm{MHz}$, and 15267 and 

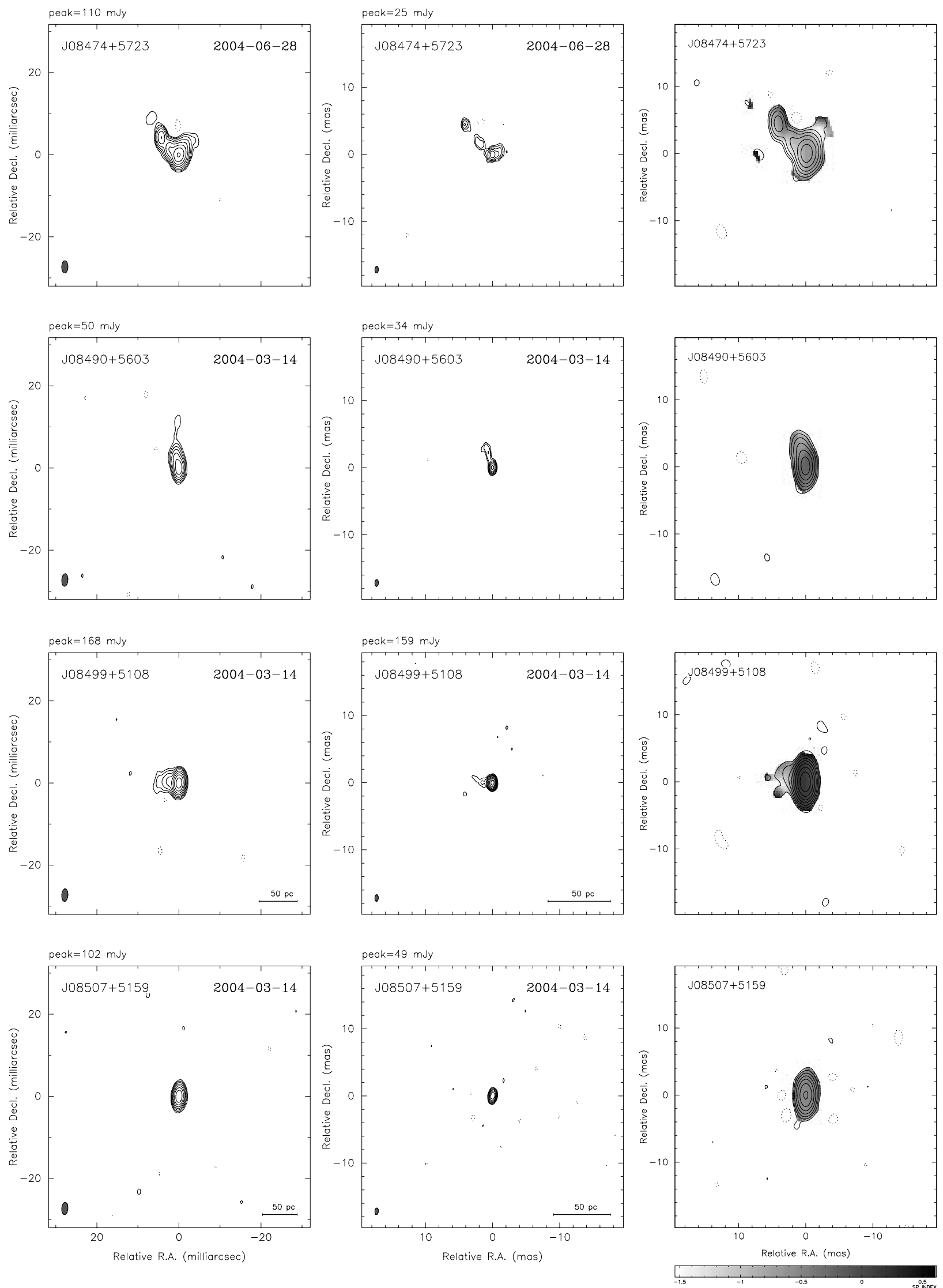

FIG. 1. - Total intensity contours of the VIPS pilot sources at $5 \mathrm{GHz}$ (left), and $15 \mathrm{GHz}$ (middle), along with spectral index (right). The $15 \mathrm{GHz}$ image and spectral index image cover the inner quarter of the $5 \mathrm{GHz}$ field. Contours start at $0.8 \mathrm{mJy}_{\text {beam }}^{-1}$ and increase by factors of 2 . Where redshifts are available, a 50 pc scale is indicated. The synthesized beam is shown in the bottom left corner. Image parameters are given in Table 2. The spectral index image is overlaid with $15 \mathrm{GHz}$ contours at a fixed resolution. [See the electronic edition of the Supplement for a color version of this figure.] 

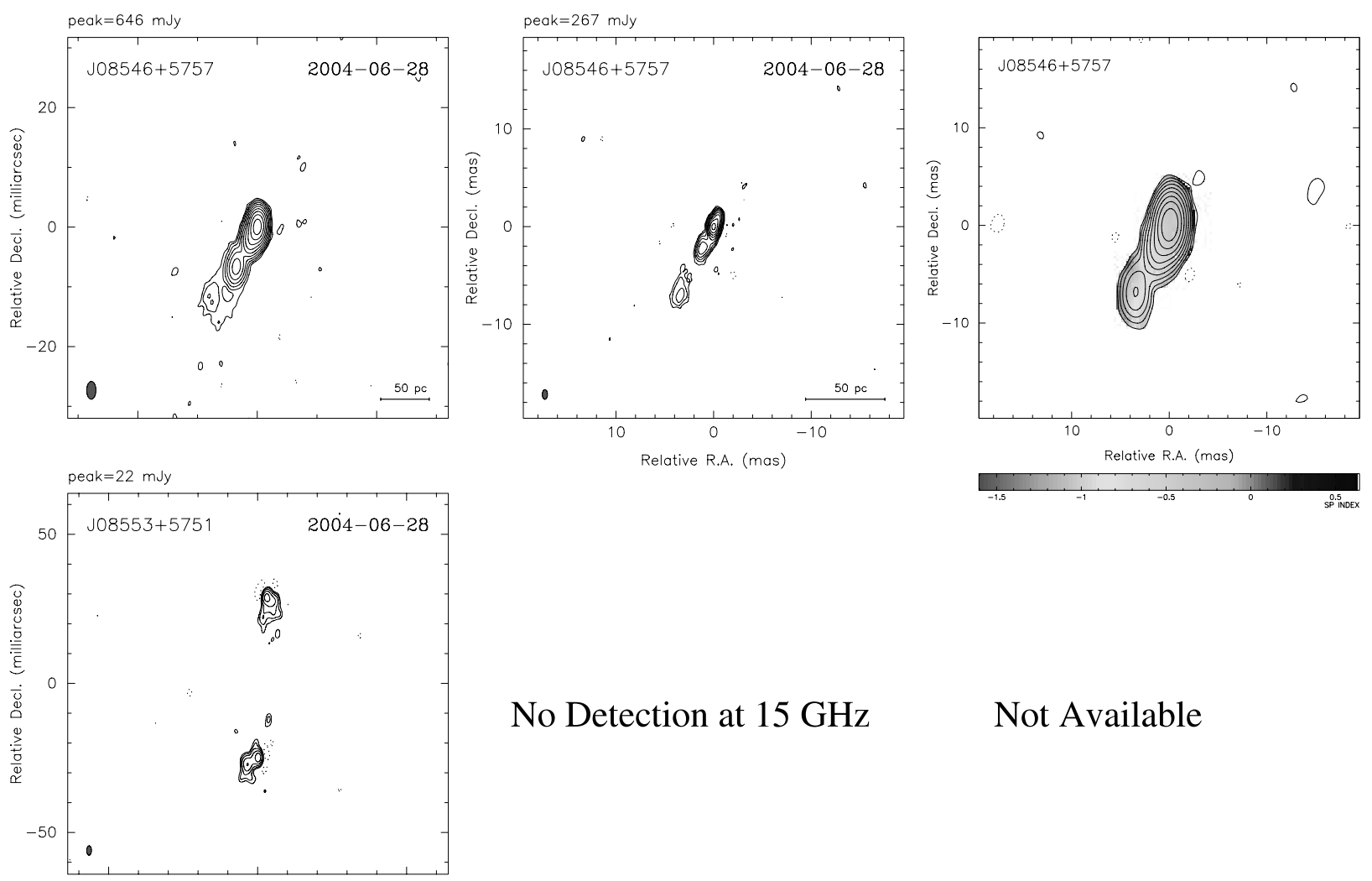

No Detection at $15 \mathrm{GHz}$

Not Available
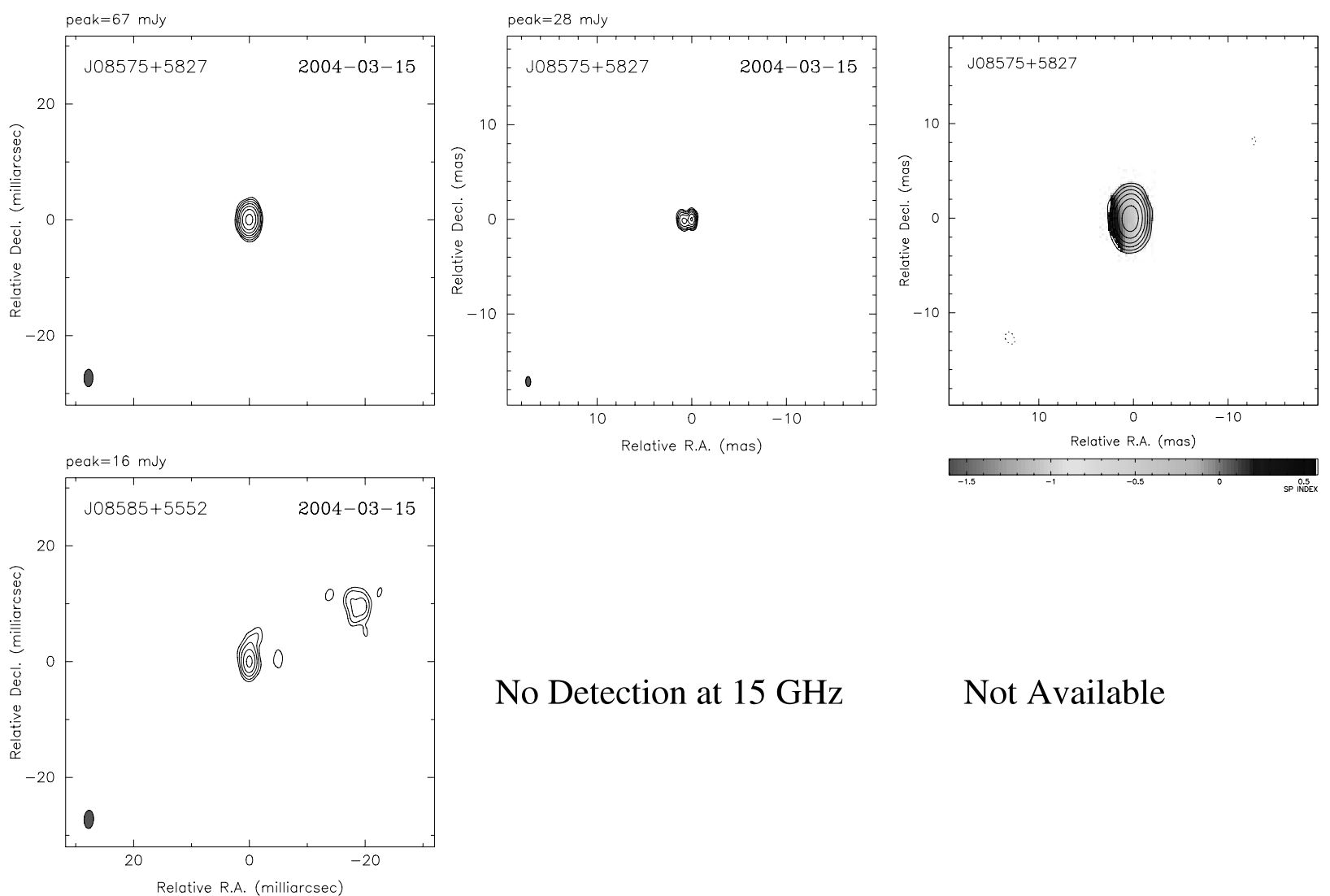

No Detection at $15 \mathrm{GHz}$

Not Available

FIG. 1.-Continued 

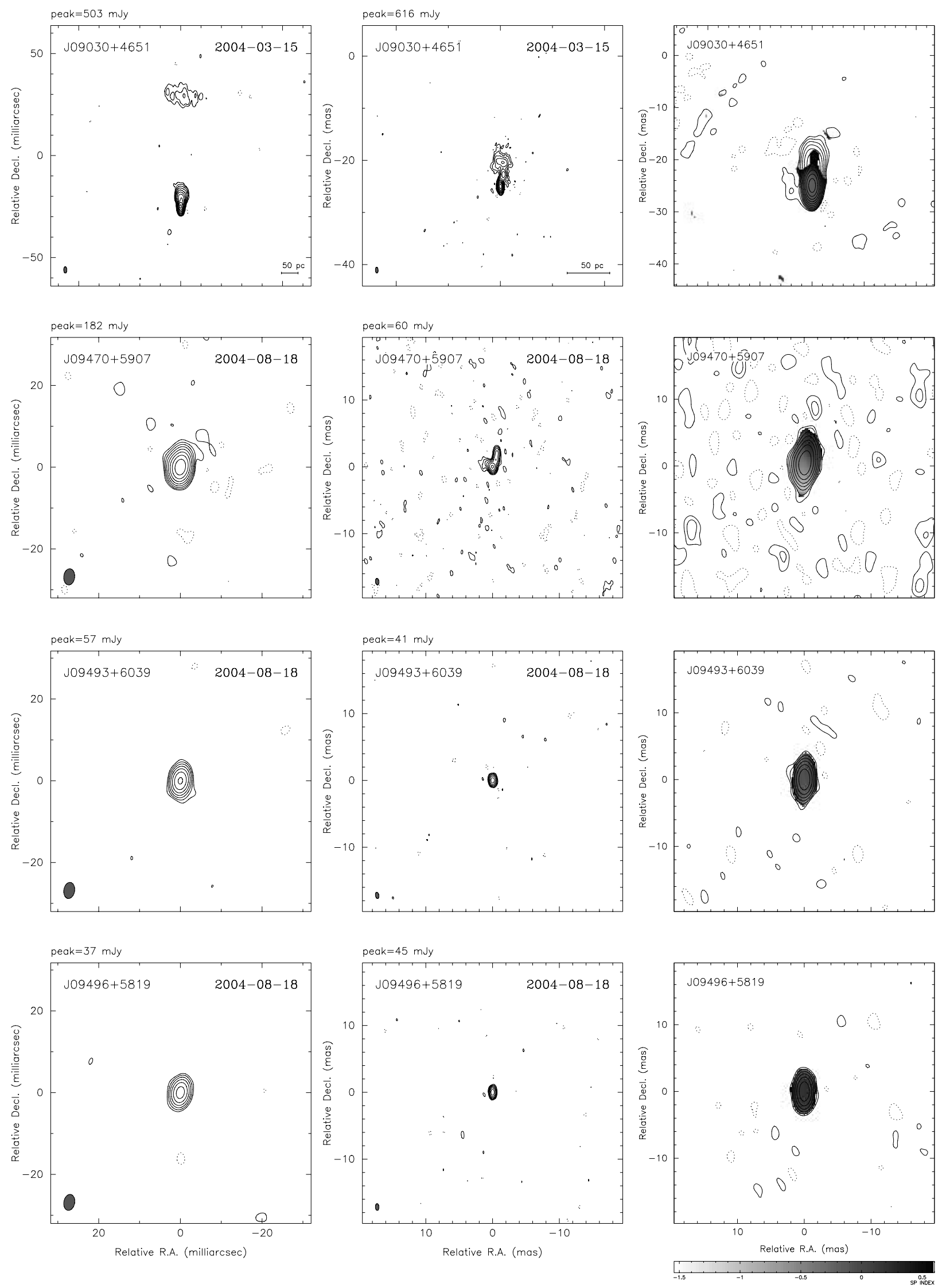

Fig. 1.-Continued 

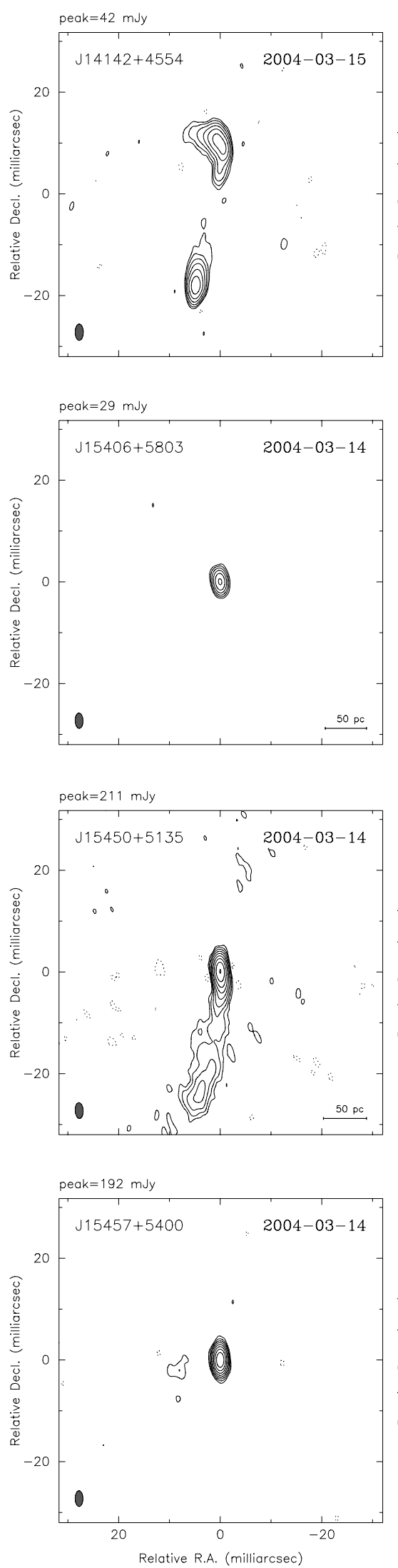

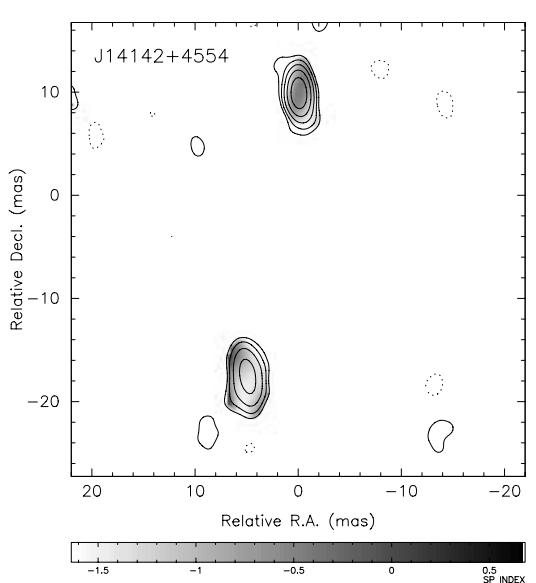

\section{No Detection at $15 \mathrm{GHz} \quad$ Not Available}
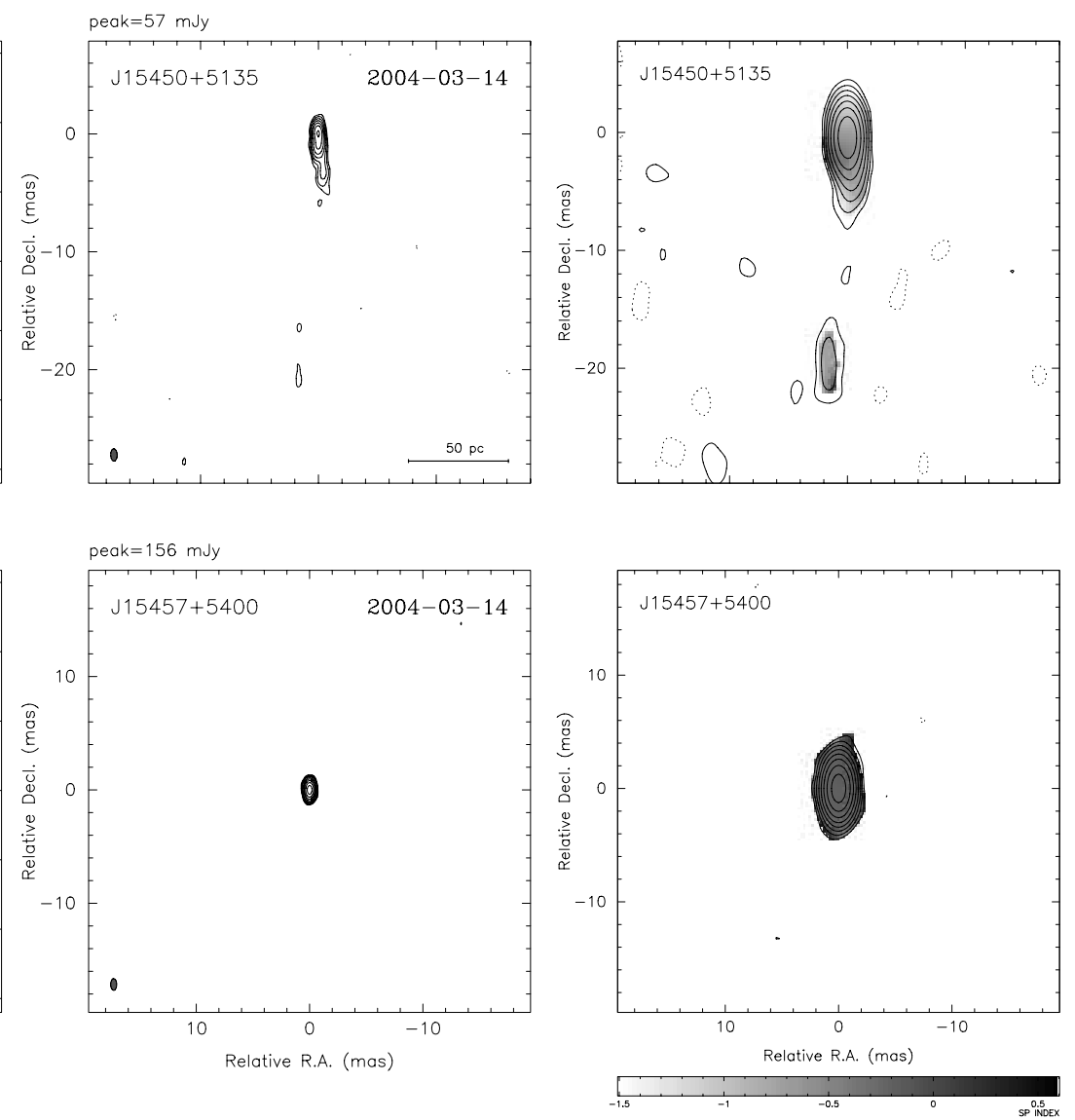

FIg. 1.-Continued 

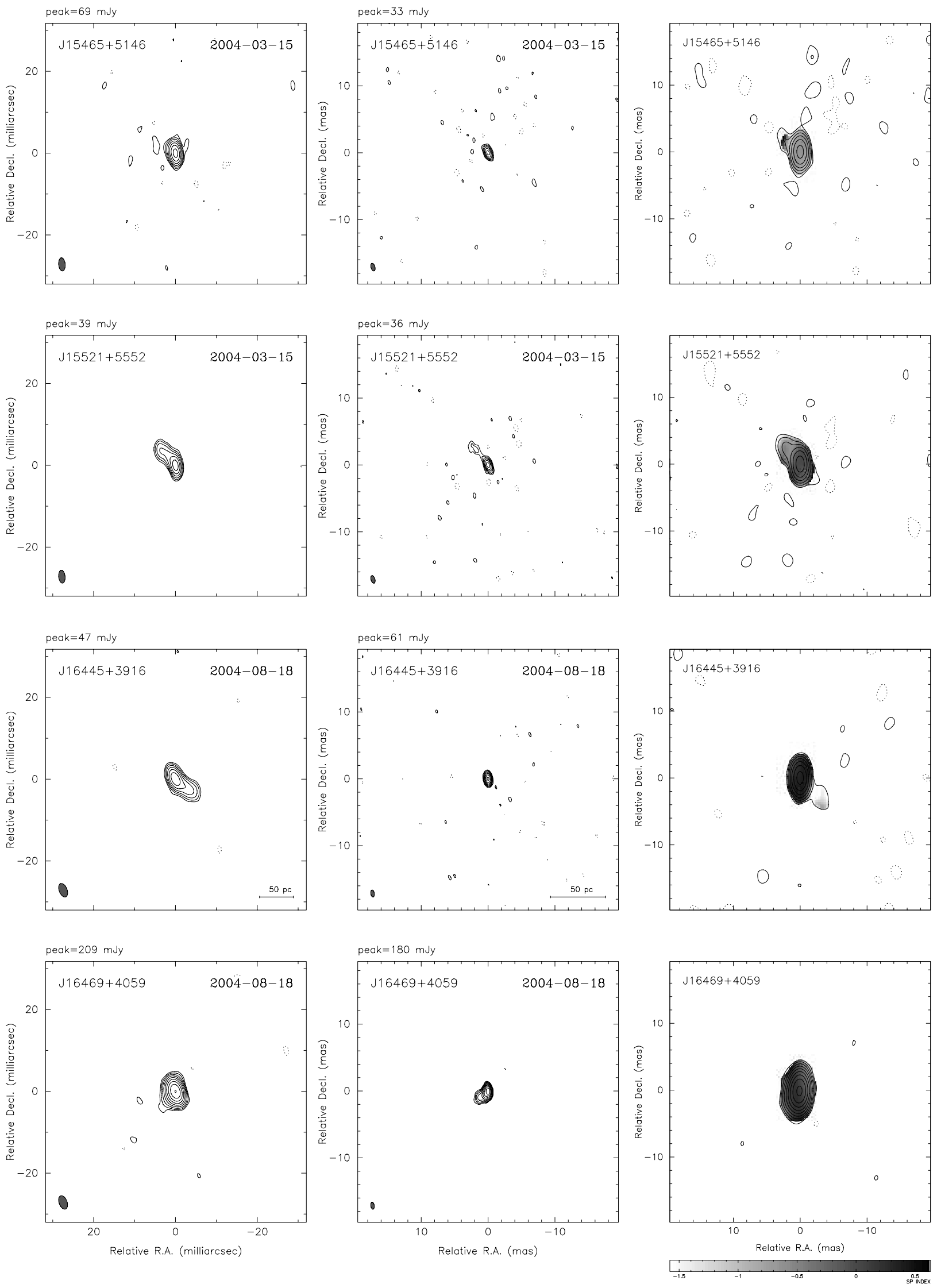

FIG. 1._Continued

34 

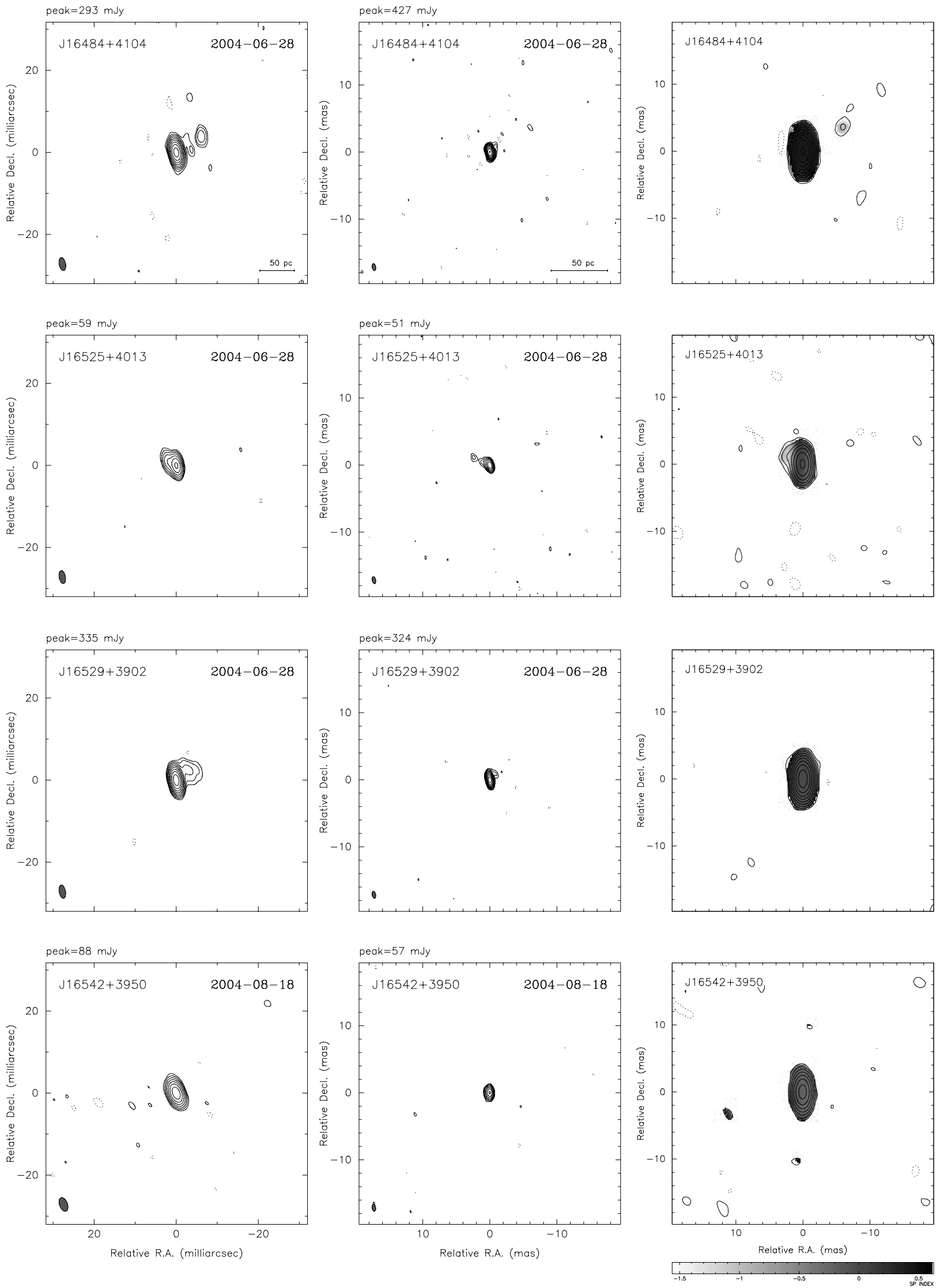

FIG. 1.-Continued

35 
TABLE 2

$5 \mathrm{GHz}$ ViPS Image Parameters

\begin{tabular}{|c|c|c|c|c|c|c|c|c|}
\hline Source Name & $\begin{array}{l}\text { Beam } \\
\text { (mas) }\end{array}$ & $\begin{array}{c}\theta \\
(\mathrm{deg})\end{array}$ & $\begin{array}{l}\text { Total Flux } \\
\text { (mJy) }\end{array}$ & $\begin{array}{c}\text { Peak Flux } \\
\left(\mathrm{mJy} \mathrm{beam}^{-1}\right)\end{array}$ & $\begin{array}{c}\mathrm{rms} \\
\left(\mathrm{mJy} \text { beam }^{-1}\right)\end{array}$ & Fit $\sigma$ & Morphology $\mathrm{y}^{\mathrm{a}}$ & Core Fraction \\
\hline J08474+5723 ............. & $2.99 \times 1.53$ & -0.8 & 219.5 & 110.0 & 0.21 & 0.998 & bj & 0.50 \\
\hline J08490+5603 .............. & $3.07 \times 1.50$ & -4.0 & 72.6 & 50.2 & 0.23 & 1.014 & $\mathrm{sj}$ & 0.69 \\
\hline J08499+5108_.............. & $3.03 \times 1.51$ & -3.5 & 191.8 & 167.5 & 0.19 & 1.009 & $\mathrm{sj}$ & 0.87 \\
\hline J08507+5159 ............. & $2.97 \times 1.50$ & -3.8 & 103.5 & 102.3 & 0.24 & 1.064 & nc & 0.99 \\
\hline J08546+5757 .............. & $2.98 \times 1.54$ & -0.6 & 875.3 & 645.8 & 0.23 & 0.979 & $\mathrm{lj}$ & 0.74 \\
\hline $\mathrm{J} 08553+5751 \ldots \ldots \ldots \ldots . .$. & $3.11 \times 1.61$ & -0.7 & 44.4 & 26.2 & 0.21 & 1.015 & $\mathrm{CSO}$ & 0.59 \\
\hline J08575+5827 ............... & $2.99 \times 1.53$ & -1.1 & 83.3 & 66.7 & 0.19 & 1.010 & $\mathrm{sj}$ & 0.80 \\
\hline J08585+5552 .............. & $3.14 \times 1.63$ & -2.7 & 30.1 & 17.1 & 0.17 & 0.995 & $\mathrm{lj}$ & 0.57 \\
\hline J09030+4651 ................ & $3.11 \times 1.54$ & 1.1 & 746.6 & 503.4 & 0.20 & 0.952 & $\mathrm{lj}$ & 0.67 \\
\hline J09470+5907 .............. & $3.96 \times 2.67$ & -6.1 & 208.2 & 181.7 & 0.29 & 0.992 & $\mathrm{bj}$ & 0.87 \\
\hline J09493+6039 .............. & $3.97 \times 2.65$ & -8.8 & 58.5 & 57.4 & 0.23 & 0.991 & $\mathrm{nc}$ & 0.98 \\
\hline J09496+5819 ............... & $3.95 \times 2.65$ & -10.5 & 37.3 & 37.3 & 0.21 & 0.968 & nc & 1.00 \\
\hline $\mathrm{J} 14142+4554 \ldots \ldots \ldots \ldots \ldots$ & $3.36 \times 1.64$ & -8.9 & 171.3 & 43.6 & 0.23 & 1.061 & $\mathrm{CSO}$ & 0.25 \\
\hline $\mathrm{J} 15406+5803 \ldots \ldots \ldots \ldots . . .$. & $3.01 \times 1.54$ & 1.4 & 35.2 & 28.7 & 0.18 & 0.990 & nc & 0.82 \\
\hline $\mathrm{J} 15450+5135 \ldots \ldots \ldots \ldots \ldots$ & $3.11 \times 1.56$ & 3.1 & 318.7 & 211.4 & 0.27 & 1.102 & $\mathrm{lj}$ & 0.66 \\
\hline $\mathrm{J} 15457+5400 \ldots \ldots \ldots \ldots \ldots$ & $3.09 \times 1.52$ & 0.5 & 192.5 & 192.4 & 0.21 & 1.036 & $\mathrm{nc}$ & 1.00 \\
\hline J15465+5146 .............. & $3.23 \times 1.59$ & 4.9 & 78.2 & 69.2 & 0.23 & 1.026 & sj & 0.88 \\
\hline $\mathrm{J} 15521+5552 \ldots \ldots \ldots \ldots \ldots$ & $3.17 \times 1.56$ & 5.6 & 59.9 & 39.4 & 0.20 & 1.000 & $\mathrm{sj}$ & 0.66 \\
\hline J16445+3916 ............. & $3.47 \times 1.92$ & 21.6 & 64.2 & 46.7 & 0.23 & 0.983 & sj & 0.73 \\
\hline $\mathrm{J} 16469+4059 \ldots \ldots \ldots \ldots \ldots$ & $3.44 \times 1.93$ & 20.4 & 247.5 & 209.3 & 0.21 & 0.985 & sj & 0.85 \\
\hline $\mathrm{J} 16484+4104 \ldots \ldots \ldots \ldots . .$. & $3.20 \times 1.55$ & 11.5 & 307.7 & 291.5 & 0.25 & 1.086 & $\mathrm{sj}$ & 0.95 \\
\hline J16525+4013 .............. & $3.20 \times 1.53$ & 9.9 & 76.6 & 59.2 & 0.21 & 0.992 & $\mathrm{sj}$ & 0.77 \\
\hline $\mathrm{J} 16529+3902 \ldots \ldots \ldots \ldots \ldots$ & $3.26 \times 1.54$ & 10.7 & 345.0 & 334.5 & 0.19 & 0.912 & $\mathrm{sj}$ & 0.97 \\
\hline $\mathrm{J} 16542+3950 \ldots \ldots \ldots \ldots \ldots$ & $3.48 \times 1.93$ & 20.6 & 91.4 & 88.5 & 0.24 & 1.017 & nc & 0.97 \\
\hline
\end{tabular}

${ }^{a}$ Source morphology is either (nc) naked core; (sj) short-jet; ( lj) long-jet; (bj) bent jet; or (CSO) compact symmetric object.

TABLE 3

$15 \mathrm{GHz}$ VIPS IMage Parameters

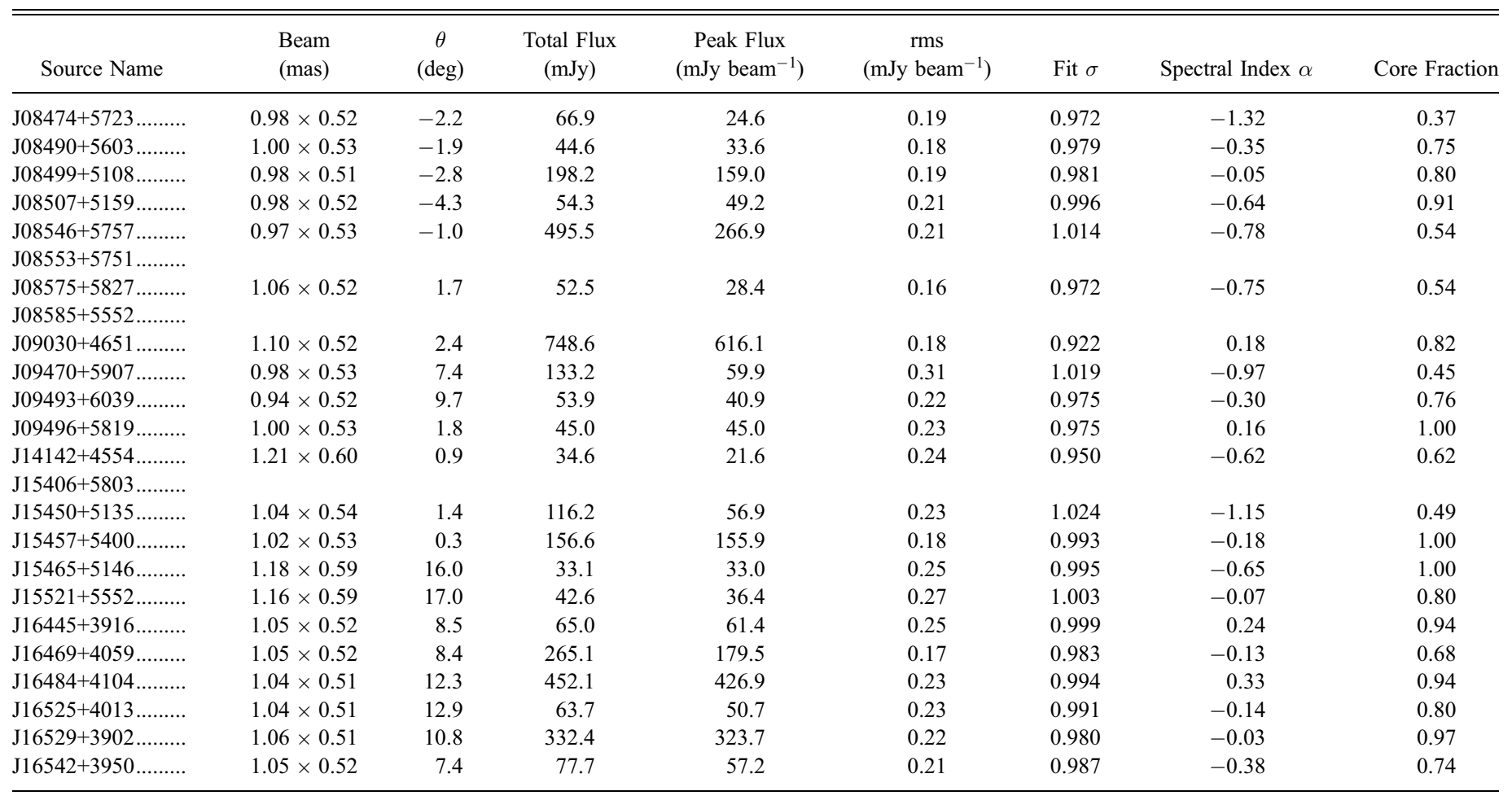



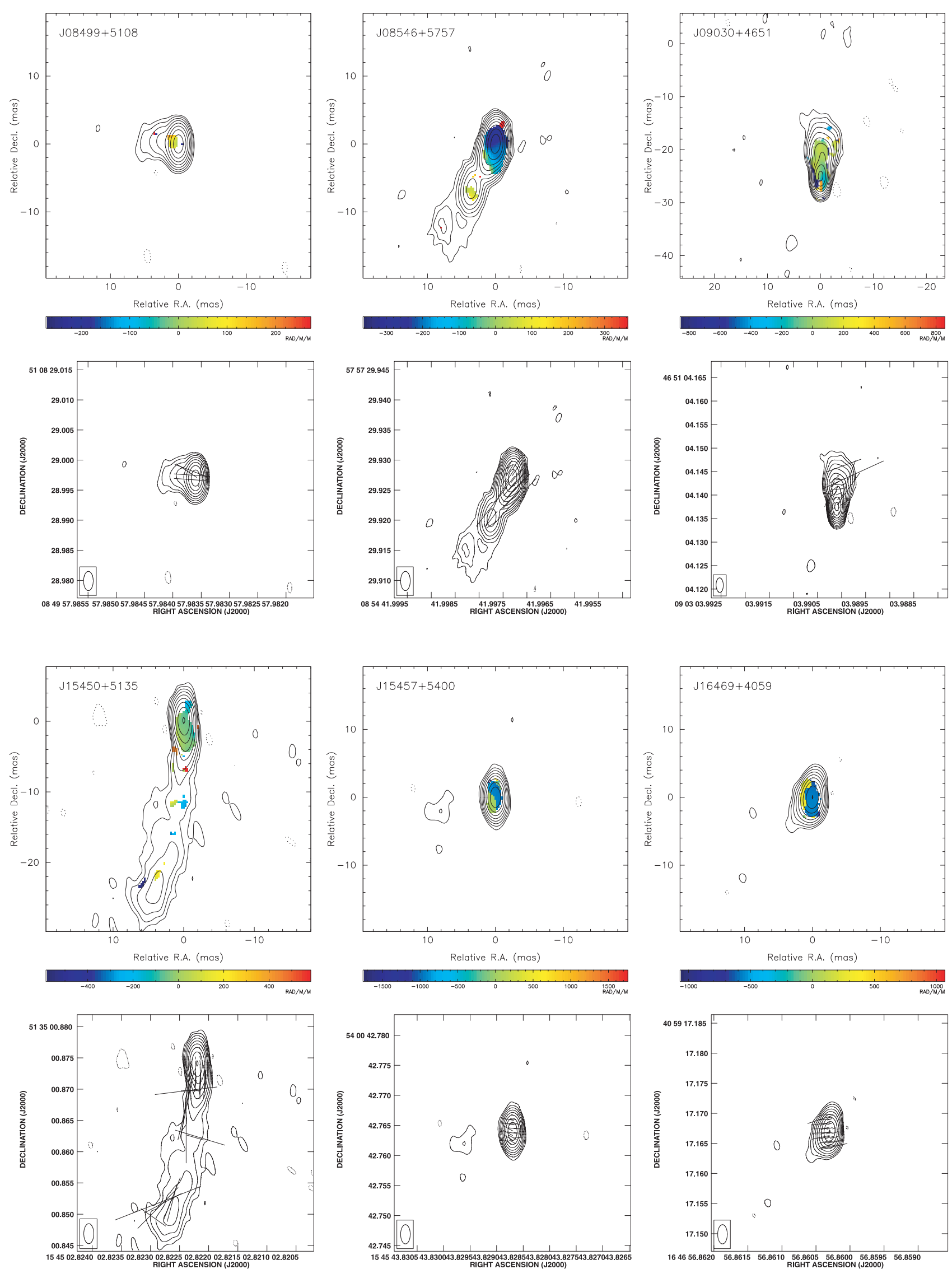

FIG. 2.-Polarization magnetic field $(B)$ vectors at $5 \mathrm{GHz}$, along with images of the rotation measure computed from polarization angles measured at four frequencies. The polarization angles have been corrected by the observed RM, and the vector lengths are proportional to the fractional linear polarization. Contours are at $5 \mathrm{GHz}$ and drawn at the same levels as in Fig. 1. 

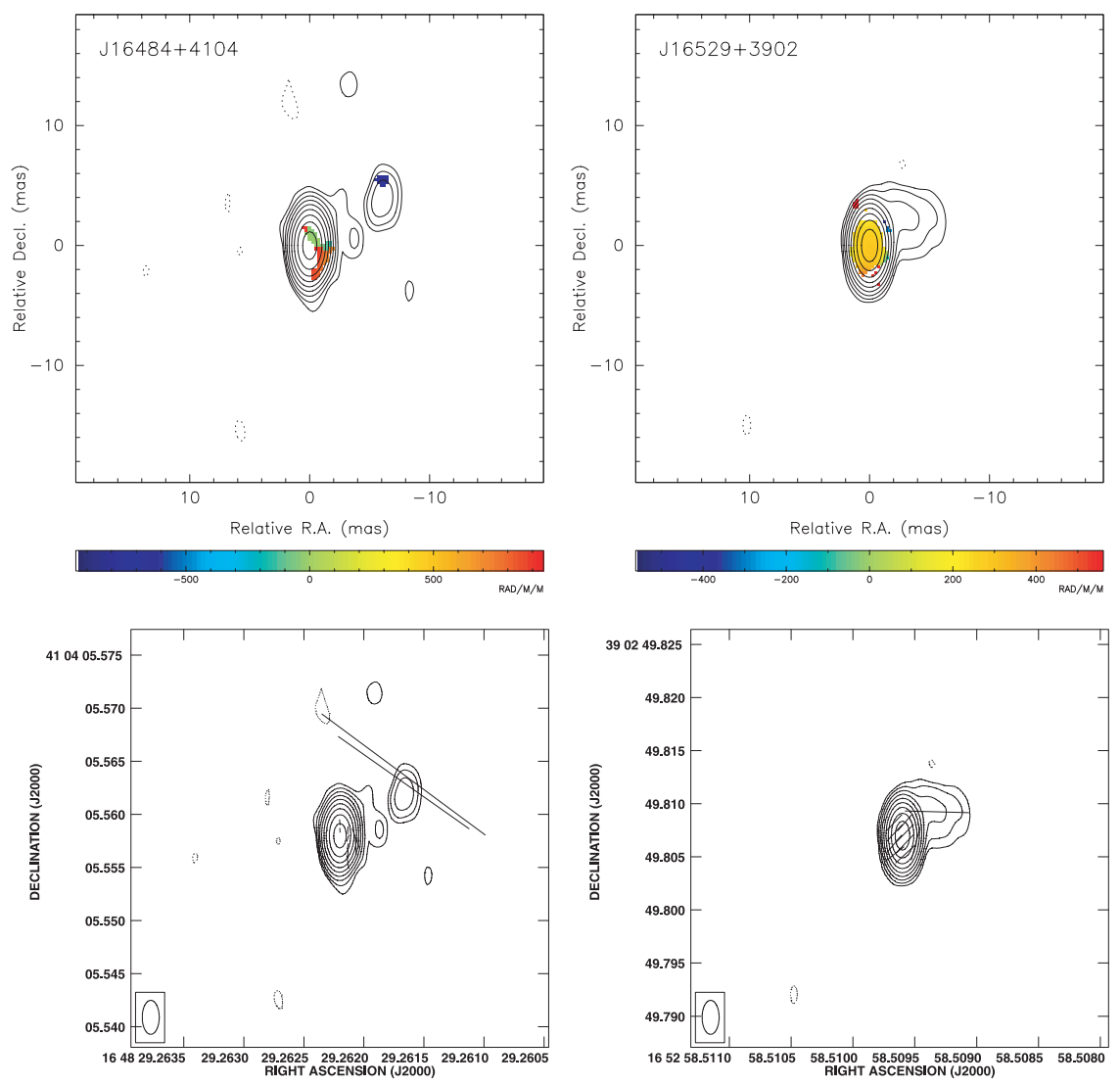

Fig. 2.-Continued

$15366 \mathrm{MHz}$. These four pairs were subsequently combined to generate images of the rotation measure by calculating the change in polarization angle with wavelength squared on a pixel-by-pixel basis. The results are shown in Figure 2, along with the magnetic field polarization vectors corrected for Faraday rotation. Under the assumption that the radiation is optically thin synchrotron emission in a homogeneous field, the vectors shown indicate the projected source magnetic field orientation.

\subsection{SDSS Magnitudes and Redshifts}

We have searched for optical counterparts for the sources in the SDSS Data Release 3 (Abazajian et al. 2005), with a counterpart defined as a catalog member falling within $1^{\prime \prime}$ of the radio position. There are optical matches for 22 of the 24 VIPS sources. The detected sources have $r$-band magnitudes between 17.5 and 22.1 (Table 1). The SDSS pipeline produces a morphological classification of "STAR" for all but three of the counterparts. These sources are almost certainly quasars, and we have designated them as "Q" in Table 1. Furthermore, SDSS redshifts are available for eight of the sources, all of which have "STAR" morphological classifications. The spectra for these sources are all typical quasar spectra, with broad emission lines of, e.g., $\mathrm{C}$ IV, $\mathrm{C}$ III, $\mathrm{Mg}$ II, or $\mathrm{H} \beta$. The redshifts range between $z=0.5$ and $z=2.0$.

\subsection{Notes on Individual Sources}

\subsubsection{J08553+5751}

The morphology of this source revealed by our $5 \mathrm{GHz}$ image (Fig. 1) resembles that of a CSO. The identification of
$0851+580$ with a galaxy, and the lack of linear polarization, is also consistent with a CSO classification, as is the lack of a detection at $15 \mathrm{GHz}$.

\subsection{2. $J 14142+4554$}

This known CSO (JVAS 1412+461) (Peck \& Taylor 2000) was included in the sample as a check on the ability of the survey to identify CSOs. In this regard the clear detection of two steep-spectrum lobes is quite satisfactory. This source has been associated with a galaxy of magnitude 19.9 and a redshift of 0.190 by Falco et al. (1998). It has a bent northern lobe and an edge-brightened southern lobe. Gugliucci et al. (2005) find no detectable motions of the lobes with an upper limit of 0.014 mas $\mathrm{yr}^{-1}$, or $0.14 \mathrm{c}$. This gives a lower limit on the age of the radio source of $2030 \mathrm{yr}$.

\section{DISCUSSION}

\subsection{Source Morphologies}

Of the 24 sources observed, we have manually classified them as 6 unresolved naked cores, 10 short jets (less than 10 mas), 4 long jets (more than 10 mas), 2 very bent jets, and 2 CSOs (see Table 2). In Figure 3 we plot the core fraction, defined as the ratio of the peak to the integrated intensity, versus the spectral index of the core component between 5 and $15 \mathrm{GHz}$. As expected, the naked core and short jet sources have the flattest spectra (with one exception of a long jet source). Such sources are likely oriented at angles close to the line of sight and are prime candidates for gamma-ray emission.

The two sources with highly bent jets have both steep spectra and a low core fraction, possibly indicating that they are 


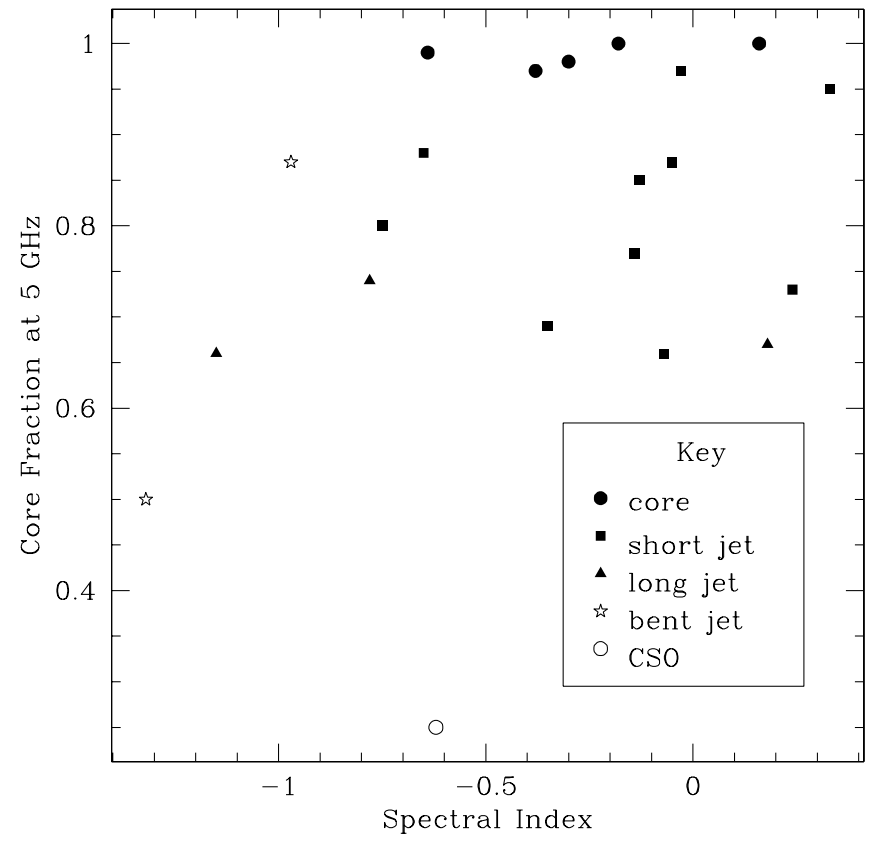

FIG. 3.-Scatter plot of the core fraction (peak intensity divided by total intensity) vs. spectral index computed between 5 and $15 \mathrm{GHz}$ for 21 sources. Sources have been classified as either naked cores, short jets (length $<10$ mas), long jets (length $>10$ mas), highly bent jets, or CSOs (see Table 2).

viewed at moderately large angles to the line of sight. In this case their sharp bends are unlikely to be due to projection effects, and must be intrinsically large. For the one CSO detected at both 5 and $15 \mathrm{GHz}$, the core fraction is overestimated since the peak in the image corresponds to a hot spot, and not to the core.

\subsection{Evolution of $A G N S$}

CSOs, by virtue of their small sizes $(<1 \mathrm{kpc})$, are thought to be examples of young $\left(10^{3}-10^{4} \mathrm{yr}\right)$ radio sources that may evolve to eventually become the well-known FRII-type radio sources (Readhead et al. 1996). Only small numbers of CSOs are known ( 40; Peck \& Taylor 2000), limiting our ability to study radio sources at early times in their evolutionary history.

We present the discovery of a probable new CSO, J08553+ 5751. Although this pilot sample is far from complete, and with only 23 sources the statistics are unreliable, the detection rate of $1 / 23$ (not counting the CSO J14142+4554 since it was deliberately selected) or $4 \%$ is interesting. Taylor \& Peck (2003) found a detection rate of $2 \%$ for sources selected from the southern part of the VCS. This is similar to that obtained for the northern part of the VCS (Peck \& Taylor 2000). As pointed out by Peck \& Taylor (2000), this detection rate is much lower than that found in the PR sample of $7 / 65(11 \%)$, or $18 / 411$ (4.4\%) found in the combined PR and CJ samples. We expect a slightly lower detection rate because the VCS is comprised predominantly of flat-spectrum sources. Nonetheless, the significantly lower detection rate found in the VCS is probably the result of the reduced sensitivity and $(u, v)$ coverage compared to the PR and CJ surveys. If the VIPS CSO detection rate is well above $2 \%$, then that indicates that the drop previously seen is not the result of looking at the fainter end of the luminosity function. With a $4 \%$ detection rate the VIPS survey would add 40 CSOs to those known, roughly doubling the number of objects. Assuming Poisson statistics apply and we get $40 \mathrm{CSOs}$, then we should be able to determine the CSO fraction to an accuracy of $\sim 0.6 \%$.

\subsection{Magnetic Fields in the AGN Environment}

Typical quasars have rotation measures that are large (>1000 $\mathrm{rad} \mathrm{m}^{-2}$; Taylor 1998, Zavala \& Taylor 2003, 2004) and time-variable (Zavala \& Taylor 2001). In the VIPS pilot sample we find core RMs ranging from $-22 \pm 45 \mathrm{rad} \mathrm{m}^{-2}$ in J16484+ 4104 to $-931 \pm 20 \mathrm{rad} \mathrm{m}^{-2}$ in J15457+5400 (Table 4). The average absolute value for the eight sources shown in Figure 2 is $390 \pm 100 \mathrm{rad} \mathrm{m}^{-2}$. This value is somewhat less than the value of $640 \mathrm{rad} \mathrm{m}^{-2}$ found by Zavala \& Taylor (2004) for a sample of 40 strong AGNs. Presumably, the Faraday screen originates in close proximity to the AGN jet, possibly created by an interaction between the jet and the ambient gas. Note that intrinsic RMs are larger by a factor of $(1+z)^{2}$. The smaller RMs in the present sample could be the result of higher source redshifts, thinner Faraday screens, or small number statistics.

Knowing the core RM value is also critical in order to make sense of the jet properties, as otherwise the RM will tend to "smear out" intrinsic relationships. With only eight sources we do not presume to look for any trends in the magnetic field orientation.

We note that the jets in five sources with extended polarization detections all have low RMs (in the range $\pm 50 \mathrm{rad} \mathrm{m}^{-2}$ ), consistent with results from Zavala \& Taylor (2004).

TABLE 4

Core Polarimetry Results

\begin{tabular}{|c|c|c|c|c|c|c|c|c|}
\hline $\begin{array}{c}\text { Source Name } \\
\text { (1) }\end{array}$ & $\begin{array}{c}P_{5} \\
\left(\mathrm{mJy} \text { beam }^{-1}\right) \\
(2)\end{array}$ & $\begin{array}{l}m_{5} \\
(\%) \\
(3)\end{array}$ & $\begin{array}{c}\chi_{5} \\
(\mathrm{deg}) \\
(4)\end{array}$ & $\begin{array}{c}P_{15} \\
\left(\mathrm{mJy} \mathrm{beam}^{-1}\right) \\
(5)\end{array}$ & $\begin{array}{l}m_{15} \\
(\%) \\
(6)\end{array}$ & $\begin{array}{c}\chi_{15} \\
(\operatorname{deg}) \\
(7)\end{array}$ & $\begin{array}{c}\mathrm{RM} \\
\left(\mathrm{rad} \mathrm{m}^{-2}\right) \\
(8)\end{array}$ & $\begin{array}{c}\chi_{B} \\
(\mathrm{deg}) \\
(9)\end{array}$ \\
\hline J08499+5108 „............. & 3.6 & 3.9 & 24 & 0.5 & 0.5 & -77 & $-282 \pm 50$ & 173 \\
\hline J08546+5757 ............. & 7.5 & 2.0 & 0 & 6.1 & 2.5 & 51 & $-259 \pm 7$ & 147 \\
\hline J09030+4651 ............. & 1.3 & 0.8 & -18 & 1.7 & 0.8 & 48 & $585 \pm 22$ & 129 \\
\hline $\mathrm{J} 15450+5135 \ldots \ldots \ldots \ldots . .$. & 0.9 & 1.9 & 74 & 0.4 & 3.3 & -70 & $-200 \pm 60$ & 28 \\
\hline $\mathrm{J} 15457+5400 \ldots \ldots \ldots \ldots . .$. & 1.8 & 1.2 & -18 & 3.9 & 3.2 & -26 & $-931 \pm 20$ & 84 \\
\hline $\mathrm{J} 16469+4059 \ldots \ldots \ldots \ldots$ & 2.4 & 1.2 & 79 & 7.1 & 3.0 & 4 & $-565 \pm 14$ & 106 \\
\hline J16484+4104 ............. & 1.2 & 0.6 & 81 & 1.4 & 0.4 & 89 & $-22 \pm 45$ & 179 \\
\hline J16529+3902 „............. & 7.5 & 2.3 & -73 & 4.7 & 1.4 & 48 & $289 \pm 8$ & 136 \\
\hline
\end{tabular}

Notes.-(Col. [1]) J2000 source name in the IAU format HHMMd+DDMM; (col. [2]) linearly polarized flux density at 5 GHz; (col. [3]) fractional linear polarization at $5 \mathrm{GHz}$; (col. [4]) electric vector polarization angle at $5 \mathrm{GHz}$; (col. [5]) linearly polarized flux density at $15 \mathrm{GHz}$; (col. [6]) fractional linear polarization at $15 \mathrm{GHz}$; (col. [7]) electric vector polarization angle at $15 \mathrm{GHz}$; (col. [8]) Faraday rotation measure; and (col. [9]) RM-corrected magnetic vector polarization angle. 


\section{CONCLUSIONS}

We demonstrate that reliable, high dynamic range, total intensity VLBA images can be obtained at $5 \mathrm{GHz}$ for $100 \%$ (24 of 24) sources with an integrated flux density of over $50 \mathrm{mJy}$ at $8.4 \mathrm{GHz}$, using a survey mode with $1.7 \mathrm{hr}$ total integration time per source. For 21 of 24 sources $(88 \%)$ high dynamic range images were also obtained at $15 \mathrm{GHz}$, allowing for the production of spectral index images. We find a third of the target sources can be imaged in linear polarization as well, allowing us to determine Faraday rotation measures. A pilot project of $48 \mathrm{hr}$ of observations yields a wealth of information for 24 AGNs. From this sample we report on the discovery of a new CSO, JVAS J08553+5751.

Future observations of the complete VIPS sample of 1000 sources will provide an unparalleled combination of size, depth, and polarization information. The large sample of high-quality data sets, especially when combined with surveys at other wavelengths, will provide an excellent resource for studying the nature of Gamma-ray loud AGNs, the jet magnetic fields, the environs of the AGN, and AGN evolution.

This research has made use of the NASA/IPAC Extragalactic Database (NED), which is operated by the Jet Propulsion Laboratory, Caltech, under contract with NASA.
Abazajian, K., et al. 2004, AJ, 128, 502 2005, AJ, 129, 1755

Beasley, A. J., Gordon, D., Peck, A. B., Petrov, L., MacMillan, D. S., Fomalont, E. B., \& Ma, C. 2002, ApJS, 141, 13

Condon, J. J., Cotton, W. D., Greisen, E. W., Yin, Q. F., Perley, R. A., Taylor, G. B., \& Broderick, J. J. 1998, AJ, 115, 1693

Dermer, C. D., \& Schlickeiser, R. 1994, ApJS, 90, 945

Falco, E. E., Kochanek, C. S., \& Muñoz, J. A. 1998, ApJ, 494, 47

Gabuzda, D. C., et al. 2000, MNRAS, 319, 1109

Gehrels, N., \& Michelson, P. 1999, Astropart. Phys., 11, 277

Gregory, P. C., Scott, W. K., Douglas, K., \& Condon, J. J. 1996, ApJS, 103, 427

Greisen, E. W. 2003, in Information Handling in Astronomy-Historical Vistas, ed. A. Heck (Dordrecht: Kluwer), 109

Gugliucci, N. E., Taylor, G. B., Peck, A. B., \& Giroletti, M. 2005, ApJ, 622, 136

Ma, C., et al. 1998, AJ, 116, 516

Mattox, J. R., Hartman, R. C., \& Reimer, O. 2001, ApJS, 135, 155

Mattox, J. R., Schachter, J., Molnar, L., Hartman, R. C., \& Patnaik, A. R. 1997, ApJ, 481, 95

Meier, D. L., Koide, S., \& Uchida, Y. 2001, Science, 291, 84

\section{REFERENCES}

Myers, S. T., et al. 2003, MNRAS, 341, 1

Pearson, T. J., \& Readhead, A. C. S. 1988, ApJ, 328, 114

Peck, A. B., \& Taylor, G. B. 2000, ApJ, 534, 90

Pollack, L. K., Taylor, G. B., \& Zavala, R. T. 2003, ApJ, 589, 733

Readhead, A. C. S., Taylor, G. B., Pearson, T. J., \& Wilkinson, P. N. 1996, ApJ, 460,634

Shepherd, M. C. 1997, in ASP Conf. Ser. 125, Astronomical Data Analysis Software and Systems VI, ed. G. Hunt \& H. E. Payne (San Francisco: ASP), 77

Sowards-Emmerd, D., Romani, R. W., \& Michelson, P. F. 2003, ApJ, 590, 109 Taylor, G. B., Vermeulen, R. C., Readhead, A. C. S., Pearson, T. J., Henstock, D. R., \& Wilkinson, P. N. 1996, ApJS, 107, 37

Taylor, G. B. 1998, ApJ, 506, 637

Taylor, G. B., \& Myers, S. T. 2000, VLBA Scientific Memo 26 (Charlottesville: NRAO)

Taylor, G. B., \& Peck, A. B. 2003, ApJ, 597, 157

Vercellone, S., Soldi, S., Chen, A. W., \& Tavani, M. 2004, MNRAS, 353, 890

Weinstein, M. A., et al. 2004, ApJS, 155, 243

Zavala, R. T., \& Taylor, G. B. 2001, ApJ, 550, L147 2003, ApJ, 589, 126

2004, ApJ, 612, 749 